\title{
THE VALUE FROM A PUBLIC HEALTH STANDPOINT OF THE DECLINING PHTHISICAL DEATH-RATE. *
}

\author{
By WILLIAM BUTLER, M.B., D.P.H.
}

OF far more importance to a people than a declining infantile mortality is the preservation of the national stamina. A multitude of weaklings is less to be preferred than a handful of virile men, and a healthy people pruned of its decadents by a high mortality amongst its children is better than a degenerate race weakened by the survival of its effete progeny. There are worse evils than premature decease, and greater blessings than a bloated population. Quality has a value, the loss of which cannot be compensated for in quantity; yet it is characteristic of the age to measure its successes by their bulk rather than by their excellence. If we estimate our hygienic progress by the number of lives saved from early death, and by the increase in the number of years lived by every million born, we shall find it satisfactory. But the aims of medicine are subserved less by the preservation of the diseased from death than they are in the establishment of health.

Instead of asking whether the total number of infants born live longer in the aggregate now than was the case, say, half a century ago, let us ask whether there is any evidence of a general lowering of the national physique and stamina, and, if so, whether this is due to the survival of degenerate types which in a less civilized state of society would have perished. If the answer to these questions be in the affirmative, we may conclude that, from the point of view of race-progress, the achievements of preventive medicine leave something to be desired and less to be thankful for. There is a common, and I think well-founded, belief that men are not now so robust as they used to be.

Defective eyesight and premature loss of hair and decay of the teeth, everywhere recognised as on the increase, may not indicate a decline of general vigour so much as a diminished vitality of certain tissues; but beyond this it is recognised that perfect health is becoming more and more the privilege of a limited few, despite the fact that the external conditions upon which it is dependent have been immensely improved. The most trustworthy evidence on this question is, however, to be got from life-tables. From

* The Presidential Address of the Willesden and District Medical Society, read, by request, before the Home Counties Branch of the Incorporated Society of Medical Officers of Health, December, 1898. 
these we discover that there has been a marked decrease in the death-rate among the young, but that among adults the death-rate has increased. I have charted a table of the Registrar-General's, showing the mean annual death-rate for males at different ageperiods in decennial groups of years from 1841-1890 (Chart I.). The direction of the curve at the top of the columns shows the increase or diminution of the death-rates at any of the age-periods given during the fifty years. It will be observed that at each age-period for males under 35 there has been during this period an almost uninterrupted decrease in the death-rate; between 35 and 45 the death-rate has remained fairly stationary; while between 45 and 75 it has increased, and above 75 again diminished. For females the increase in the death-rate at the higher age-periods has not been so marked, nor does the increase appear until a greater age has been attained. This increase in the rate of mortality among adults means one of two things: either that the average stamina of the population is diminished, or, if their stamina is unimpaired, that the external conditions of life are now more injurious to the vitality of adults than they were half a century ago.

Neither alternative is pleasing, for in either case it means that the national stamina has not kept pace with the demands made upon it, that the adult population of to-day is less fitted to adapt itself to its environment than it was in the days of our grandfathers. It is sometimes stated that " the same causes which have lowered the mortality at the earlier ages have also improved the average health of those who survive" (Newsholme). This by no means follows. The causes which have lowered the mortality at the earlier ages have told more upon women than upon men in increasing their expectation of life, but the average health of women is usually worse than that of the sterner sex. Proverbially, the rickety gate swings longest, and the weakly constituted in civilized society receive within limits their stamp of fitness from this very defect. Shielded as they are from the more trying struggles and hardships to which the stronger members of a community are exposed, they frequently survive a greater number of years, albeit of ill health, than their physically more favoured competitors for existence; and this increased survival of those who cannot be said to enjoy health at all lowers the average of the health of the community. That the diminished mortality-rates from certain diseases once so fatal in early life have resulted in the survival of a greater number of weakly-constituted persons is shown by the increased rates of mortality from eertain other diseases fatal in adult life. From tables prepared by the Registrar-General, I have charted 


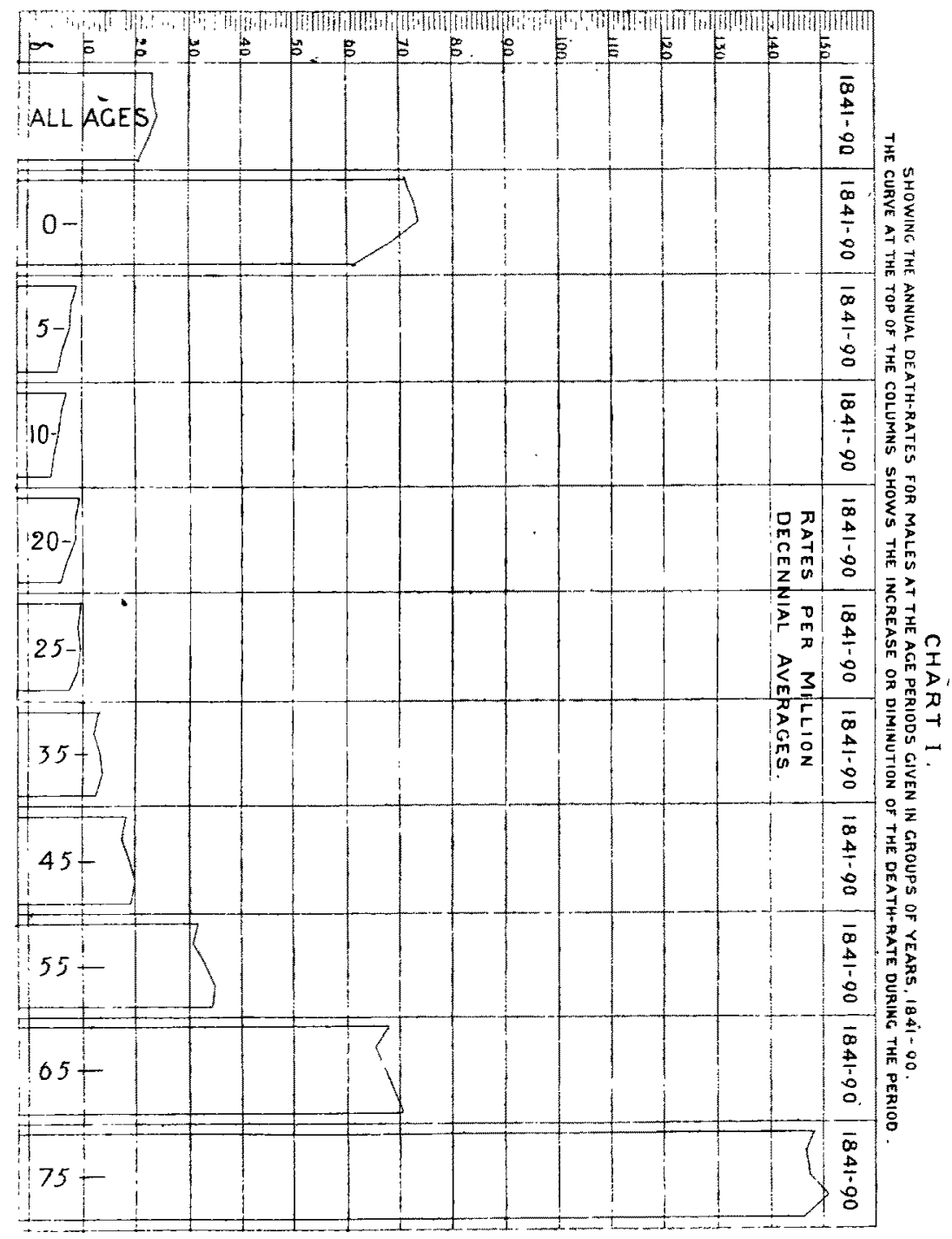




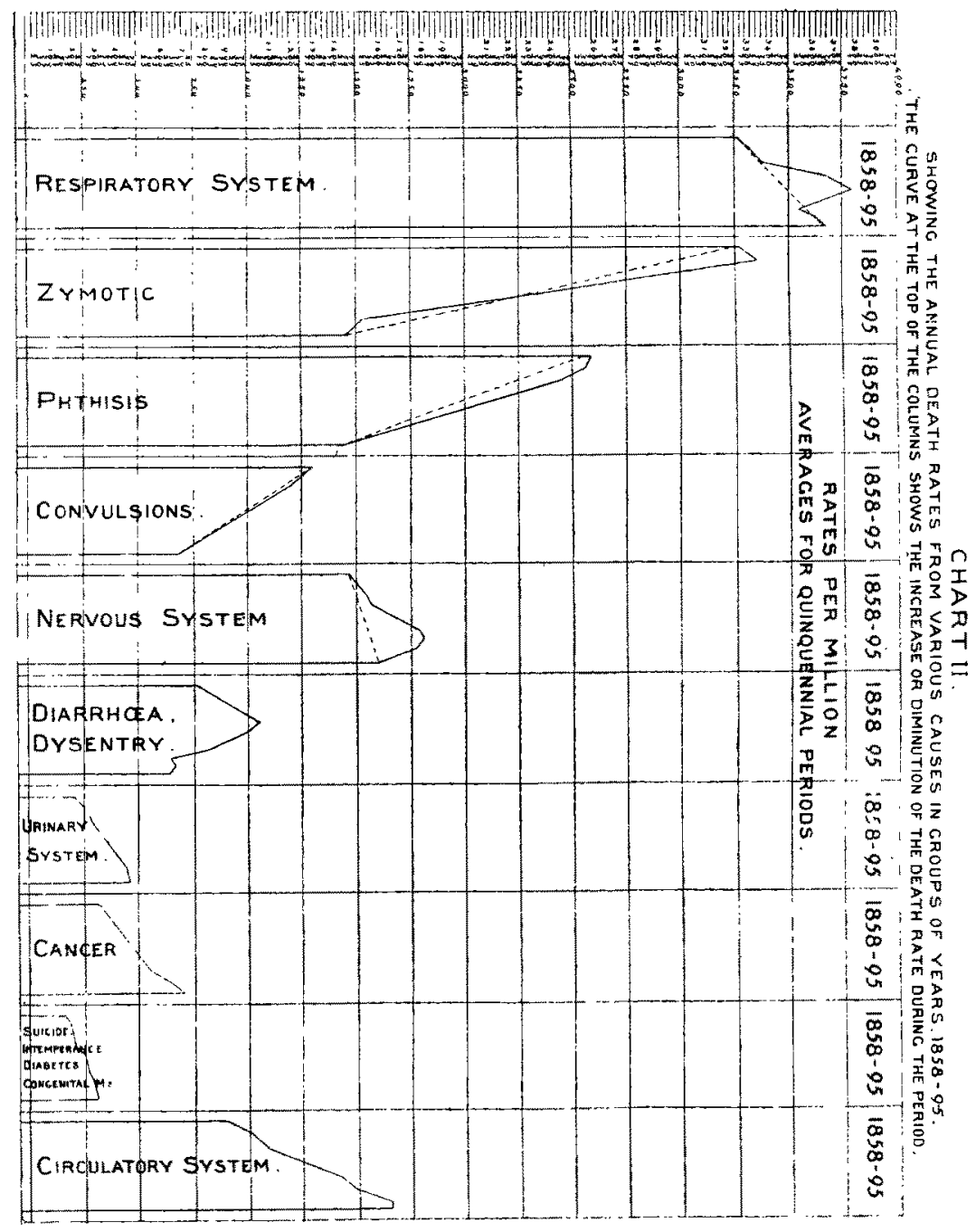


the death-rates from certain diseases and groups of diseases, the mortality-rate from which during the last forty years has shown a marked tendency to increase or decline, and the approximate correctness of which as indicating what is actually occurring is generally admitted. From this chart it will be seen that zymotic diseases, phthisis and convulsions have a death-rate which has progressively and rapidly declined during this period, while that from diseases of the circulatory, urinary, respiratory and nervous systems, and from cancer, has markedly increased. Suicide, diabetes, premature birth and congenital malformations, rickets, anæmia and intemperance, also show an increased death-rate, the significance of which I shall refer to later (Chart II.).

It is my purpose in this paper to indicate some of the effects which, from our knowledge of phthisis, we should infer would follow the decline of its death-rate, and to present such evidence as appears to demonstrate the correctness of these inferences.

Disease is conditioned as much by innateness in the organism as by pathogenic agents in the environment. The pathogenicity of micro-organisms exists solely because of the susceptibility of their hosts, and this susceptibility is as much a cause of disease as is the existence of the pathogenic microbe. Where susceptibility to a disease is an inheritance common to all-as, for the most part, it is in the case of zymotic diseases-this is less obvious than where, immunity being the rule, susceptibility becomes an individual morbid proclivity. Thrown into relief by its exceptional character, the liability to a disease is then seen to be a constitutional peculiarity of the individual, and as such becomes a condition of the disease as obviously essential as any which may be labelled pathogenic. If it were possible to classify people by their persistent morbid proclivities in addition to classifying the extra-constitutional conditions on which the manifestation of their diatheses is dependent, we should establish data which, from the point of view of preventive medicine, would be second in importance to no other.

For instance, in dealing with phthisis, we say it is produced by infection with the tubercle bacillus. Predisposing conditions of infection are damp subsoil, overcrowding, insufficient ventilation of living-rooms, and generally those conditions which are summed up as bad hygiene. The immediate causes of infection are ingestion or inhalation of the tubercle bacillus by drinking the milk and eating the flesh of tubercular cows, or inhaling dust containing dried particles of tubercular sputum. That this is but a partial statement of the causation of phthisis is seen when we observe that not all people living under the conditions just stated 
become subject to pulmonary tubercle. The majority of people so living do not in fact develop the disease, and those who do, if we know their previous elinical and family history, are those whose fate we can predict with tolerable accuracy. For the most part they exhibit no redundancy of trophic energy; they are spare; their tissues are ill-nourished; their chests are narrow and frequently misshapen, and they have throughout life been delicate, liable to catarrhs, anæmias, and other minor disturbances of health. Their family histories for the most part are bad. Numerous relatives have died of phthisis, others have suffered from gout or rheumatism, not infrequently there is a history of insanity, epilepsy, alcoholism and other neuroses. It is manifest that, in addition to the unfavourable environment which has been observed as a contributory condition of the disease, our phthisical patients bring another equally if not more important in the bad stock, which is almost as invariably an antecedent of the disease as are the insanitary conditions which favour infection.

That phthisis is rarely, if ever, transmitted by direct inheritance, and that it is dependent for its development upon certain recognised external conditions, is of less significance than the fact that susceptibility to tubercular infection is found in families the different members of which, generally speaking, display in addition to this particular morbid proclivity other not less important signs of degeneracy. I say of less significance, because I believe that commonly the vulnerability of the lung or other tissue to tubercle is only one of a series of inherited structural and nutritional peculiarities which constitute an organic degeneration inherent, deepseated and widespread.

"Perhaps the disease most largely found in neurotic families beyond their neuroses is phthisis," says Dr. Clifford Allbutt " Visceral Neuroses"). "In case after case I find phthisis in parents or kin. . . F For many years I have noted the frequent coincidence of phthisis and of rheumatic fever in family trees, and I think this coincidence has been observed by others. To my mind, then, the natural group is neurosis, phthisis, and acute rheumatism. But what of the gout? Rheumatism and gout are certainly akin, the two maladies appearing in the same families, and even in the same persons, at different ages. Yet I think gout goes by a different route to its direct work. . . . Although there is no eross between neurosis, phthisis and gont, yet one may trace the history of gout upwards to a point of union with a divergent stock, which two, three, or four generations back had branched off into the tuberculoneurotic." 
Dr. Clouston, in his lectures on the Neuroses of Development, says: "I had in 1863 shown the close connection of lung tuberculosis and insanity, hereditarily and personally. It is very common to find the two diseases in different members of the same family, and there is every reason to suppose from the facts that a heredity towards phthisis may determine insanity, and vice versâ. The percentage of deaths from tuberculosis is four times higher among the insane than among the general population at the same ages. . . Assuming, as I think we are entitled to assume, that the low nutritional condition of the body is the great predisposing cause for tubercular infection, I contend that this innutrition is one form of trophic neurosis, and that the special pulmonary innutrition that permits of the tubercle bacillus finding a nidus in the lungs probably results from a trophic developmental failure in the epithelium of the alveoli of the lungs, in consequence of which the bacilli find free entrance and a suitable nidus there. Its connection with insanity hereditarily shows the affinity of the trophic failure to the highest of all the neuroses. The coincidence of the maximum age of tubercular lung infection and of greatest liability to one of the most important of the acquired insanities, that of adolescence, is a very striking fact, and, taken together with the facts as to heredity, seems to show that if tuberculosis cannot itself be called a neurosis, it is in most cases dependent for its existence on a trophic neurosis, or has the closest affinity to it."

These are facts to be borne in mind when we speak of the decline of the phthisical death-rate as a matter for unequivocal congratulation. Phthisis, as will be seen from the chart, is responsible for a death-rate of only two-thirds of what it was less than half a century ago, and if the same rate of decrease be maintained, the next century will see the British as free from phthisis as they are from leprosy. In round figures, there are saved each year 1,000 lives out of every 1,000,000 living in England and Wales from this disease alone, assuming that, but for the accomplished sanitary improvements of the period, the death-rate of half a century ago would still have been maintained. But we must remember that mankind has its decaying branches, which are preyed upon and exterminated by parasitic organisms, whose attack the strong and vigorous successfully resist. The fungi infesting the dying bough of a tree, the sturdy living branches of which resist their assault, exhibit a natural process which cannot be looked upon as other than beneficent; and a rigid system of asepsis, which protects a degenerate species from the incidence of its natural enemies, may react detrimentally upon the race by preserving an undesirable type which 
would otherwise have been destroyed. It is because the external conditions necessary for the development of phthisis have been modified-not because the phthisically - disposed, the degenerate offshoots of the human family, have coincidently decreased-that we are able to record a diminished mortality from the disease. It may, of course, be asked how far this decline is to be ascribed to improved sanitary conditions alone, and how far it is due to modifications of those innate morbid proclivities which, predisposing to phthisis, to neuroses, to rheumatism, and to gout, receive a determining bias from other conditions in the environment than the merely hygienic. In other words, has not civilization in its march modified the temperaments and the diatheses of people, so that, in the case of the diathetic group we are considering, the morbid proclivity is becoming more neurotic and rheumatic, and less tubercular and gouty? May not the phenomenon of replacement of one neurosis by another in the individual have its analogue in replacements of the more differentiated nouroses in different members of a neurotic stock? I have known patients who suffered from megrim lose their megrim and become subject to epilepsy, and vice versâ. One patient for whose migraine I had prescribed became subject to asthma and hay-fever, and lost her migraine; another, who had suffered severely from migraine most of her life, ceased from having attacks when she became the victim of renal colic. An operation in which a large renal calculus was removed was followed by recovery from the renal colic and a recurrence of the migraine after some years of freedom from the attacks. A dipsomaniac became insane, and after recovery in an asylum from her insanity found that she had lost all inclination to partake of stimulants, but was subject constantly to auditory hallucinations, in which she heard voices prompting her to remain abstinent, while at the same time she became intensely religious. Trousseau's classical case, in which the famous physician cured his patient of megrim, only to see him fall a victim to the gout which put a period to all his sufferings, will recur to you in this connection. In some degree an analogous replacement is to be observed in regard to the more individualized neuroses, the replacement occurring in different members of a family instead of in the same person. In the same family we find one member suffering from phthisis, another subject to acute rheumatism; in another branch of the family there is gout and a liability to functional neuroses. We may distinguish one diathesis from another, while recognising their co-existence in the same individual, and readily conceive that the conditions which determine the development of the one diathesis at the same time 
produce a relative immunity to the development of the other. Sir William Broadbent (Lancet, October 29th, 1898) observes " that gout seems to reinforce the resistance to infection by tubercle; and, curiously enough, so does the anæmia to which young girls are subject." Yet we know that gout and phthisis and anæmia run hand in hand in the same family.

We need not pursue the speculation further, since, whether the decline of phthisis proceeds from purposive hygienic modifications of environment or results from changes incidental to the altered habits and pursuits of the age, the effect is the same.

The coincidence of two or more diatheses in the same person or group of persons, one of which is fatal before or during the procreative age, and the others not, must inevitably result, if the development of the fatal condition is turned, in an increased survival of those persons subject to the correlated diatheses. Whether we ascribe this decline in a death-rate fatal to neurotics, to social or to hygienic causes, or to both, the practical bearing of the fact remains the same. If we are to have a people sound in body and in mind, evidently it will not do to modify environment alone. It is conceivable, that if all people now living were placed under what is at present considered a perfectly healthy environment, the result would not be the acquirement of perfect health, but rather the ultimate production of a race in which every individual was a physical degenerate. More than this, it may be said that every modification of environment which permits the less vigorous of a species to survive is a possible step towards degeneration, and that unless the reproduction of those with persistent morbid proclivities be checked every triumph of science which enables them to prolong their existence and multiply their like has a retrograde effect, which can only'culminate in the ultimate physical, if not moral and mental, degradation of the race.

As we have seen, the bacillus of tubercle selects as its victims those showing hereditary morbid proclivities which it is highly undesirable should be perpetuated-morbid proclivities which are not confined to a special vulnerability to tubercle, but include a variety of conditions which, while they are less fatal to the individual, are on that account the more disastrous to the race. The diminished mortality from tubercular diseases would lead us to expect an increase in the incidence of the other less fatal diseases to which those inheriting the tubercular diathesis are specially predisposed. Thus, if tubercle preys specially upon the neurotic, we should, ceteris paribus, expect an increase of neuroses. Evidence of the increase of these is for the most part impossible so far as 
vital statistics are concerned, yet we hardly need the assurance of vital statistics for the common belief that neuroses are on the increase.

If we consider for a moment what those morbid conditions are to which the tuberculo-neurotic are subject, we shall see that, while they constitute a class of diseases the increase of which available statistics must fail to demonstrate, they are, at the same time, perhaps the most common ailments which the general practitioner is called upon to treat. Without attempting an exhaustive list, I will mention some of the more typical conditions to which the tuberculo-neurotic are specially subject. They are: Insanity, epilepsy, hysteria, migraine, asthma, and angina pectoris; the visceral neuroses, such as gastralgia, enteralgia, and dysmenorrhoea; the neuroses of the cerebro-spinal nerves, such as sciatica, pleurodynia, and tic-douloureux. In addition, we must include the structural defects so constantly associated with the diathesis. In the eye these are hypermetropia, myopia, and, above all, astigmatism, an asymmetry of the eye which accords with the known tendency of neurotics to other asymmetries, notably of the face, head, and hard palate. A special liability to anæmia must be noted, with innutrition of the tissues generally, and a predisposition from this cause to uterine flexions and cervical and perineal lacerations during parturition.

The psychical characteristics which accompany the neurctic diathesis constitute one of the gravest aspects, as well as one of the most striking evidences, of the increase of neuroses. The neural instability which in the case of the lower centres shows itself in an attack of asthma, laryngismus, or megrim, betrays itself in the case of the higher centres in morbid sentimentality, crankiness, and faddism. The neurasthenics, hypochondriacs, anti-vivisectionists, anti-vaccinationists, Contagious Diseases Acts abolitionists, egomaniacs-designate them by what symptom you will-constitute a class of intellectual or emotional neurotics who are organically related to those who in another age would have been largely eliminated by the ravages of tubercle. These different manifestations are not, of course, present in every individual neurotic, nor can we say that every neurosis is the outeome of a nutritional defect which in all cases predisposes to phthisis, but the neurotic and phthisical diatheses, as we have seen, are usually combined in the same individual, and it is easy to see that an increase of the emotional as well as other neuroses should follow the increased survival of those inheriting the combined diathesis. The other most intimate associate of the tuberculo-neurotic group is acute 
rheumatism, with its sequelæ, cardiac valvular disease, chorea, embolism, etc.

Now, although it is impossible to demonstrate the increase of all these morbid conditions, in the case of some we have statistical evidence which points strongly to the conclusion that extension has occurred. First let us take the insane, whose predisposition to tubercular disease is undisputed. From a table in the fifty-second Annual Report of the Lunacy Commissioners we find that since 1859 there has been a progressive increase in the number of officiallyknown lunatics. Thus, in 1859 the number of officially-known lunatics to each 1,000 of population was $1 \cdot 8$; in 1869 it was $2 \cdot 3$; in $1879,2.7$; in $1889,2.9$; and in $1898,3.2$. It is only fair to admit that these facts do not warrant the conclusion that there is necessarily an increase of occurring insanity. For not only is there in these days a greater survival and consequent accumulation of the insane, but the number of officially-known lunaties bears now a much greater proportion than it did to the total lunacy of the country. On the other hand, it should be noted that, despite the fact that a greater proportion of the insane are cared for in asylums, the suicide-rate has progressively increased. This is somewhat analogous to the fact that, while the drinking habits of the nation have immeasurably improved, inebriety has increased, and that while the death-rate from diseases of the liver has declined, that in which the deaths are ascribed directly to intemperance is growing. Drug manias are also spreading in a startling manner, and all the facts appear to indicate an increase of the type prone to insanity.

I have already spoken of the association of refractive errors with the neurotic diathesis. Now, errors of refraction are admittedly more common than they were. The increasing number of children who have to wear glasses is a convincing proof of this, although statistical evidence of the fact-in this country, at least-is wanting. Doubtless this increase is partly an acquired defect from the increased eye-strain which modern life entails, but the greater proportion of dioptric defects are hereditary, if not congenital. Sometimes these refractive errors are said to be the cause of the neuroses, and there is no doubt that many neurotic manifestations, such as epilepsy, migraine, and hysteria, are aggravated by the oculoneural irritation arising from these errors. While admitting, however, that they aggravate a neuropathic proclivity, I believe that they are more concomitant manifestations than antecedent conditions of the different neuroses with which they are associated. Dr. G. T. Stevens, in his book on Functional Nervous Affections 
(New York, 1897), very well states the relationship in the following three propositions :

1. "Hereditary neuroses, such as epilepsy, migraine, neuralgia, chorea, and insanity, and the same principle may be stated to hold in respect to phthisis, are not transmitted from parent to child directly.

2. "Such neuroses are the manifestations of transmitted physical peculiarities which render difficult the performance of certain important functions.

3. "That of the hereditary physical defects which thus tend to develop neuroses, anomalous conditions of the eyes are among the most frequent and important."

Of sixty patients who consulted me for functional nervous disturbances of a persistent and severe character, whose eyes I examined, 10 per cent. were emmetropic, $21^{\circ} 6$ per cent. were hypermetropic, $63 \cdot 3$ per cent. were astigmatic, and 5 per cent. only were simply myopic. Of the astigmatic cases 26.6 per cent. were of mixed, and $18 \cdot 3$ per cent. of myopic astigmatism. Anisometropia was present in about 52 per cent. of the cases. These figures are in contrast to what would be experienced in ordinary ophthalmic practice, for the reason that the examination of the eye was undertaken primarily on account of the neurotic symptoms about which I was consulted.

In the light of facts such as I have just mentioned, the admitted increase of refractive errors is of profound significance as an index of the effect of a diminishing mortality from phthisis not due to decline of the hereditary causes.

A death-rate is much more valuable as an indication of the prevalence of a disease if that disease is ultimately fatal, as in the case of cancer, and, to a large extent, of phthisis, than if a fatal termina. tion is the exception, as in chlorosis, acute rheumatism, or epilepsy. At the same time, if, in spite of improved methods of treatment, a death-rate from a disease is increasing, we shall be justified in concluding that it is in itself ground for believing either that the disease has assumed a more virulent type, or that it represents an increase in the relative number of people attacked. I shall only point out in this connection that the death-rates from anæmia, acute rheumatism, and valvular diseases of the heart have increased considerably during the period which we have under review, that from the Registrar-General's group, anæmia, leucocythæmia and chlorosis, having doubled itself in twenty years.

On the other hand, the death-rate from epilepsy and convulsions has conspicuously diminished, and at first sight this would appear to contradict the view that the decline in the death-rate from 
tubercular diseases has been associated with an increase of those liable to other diseases to which the tubercular diathesis predisposes.

It must be remembered, however, that epilepsy in itself is not a fatal disease, and the increased care with which epileptics are now attended has enormously reduced the risks to which their malady exposed them. The decline in the mortality-rate from infantile convulsions-so far as it represents any real diminution of deaths from this cause-is on a par with that from phthisis, in that it is an index of increased survival of those predisposed to convulsive seizures. Every source of irritation to the infantile nervous system which may manifest itself in reflex phenomena is now rigidly sought for and removed. The child which would have died from convulsions a quarter of a century ago, because of an instability of its nervous centres incapable of withstanding the irritation arising from a long foreskin, an error of diet, the presence of ascarides, etc., runs now an infinitely better chance of survival on account of the greater care with which such causes of irritation are removed; but this is only another factor which is contributing to the survival of children with a higher average nervous instability than was consistent with survival in the past.

But, at the best, the evidence culled from statistics is only a laboured attempt at measuring in definite directions different aspects of a fact the acceptance of which common experience has sanctioned. That nervous affections, particularly of a functional type, are becoming increasingly common is beyond dispute. Usually their increase is associated with that of the wear and tear, strain and worry of civilized life; but, even so, it is upon those who inherit a lowered vitality, on those who exhibit an inherent proclivity to morbid function or to organic decay, that this increased stress is operative. In the increased rate of mortality from congenital malformation and premature birth we may trace an effort of Nature to check the reproduction of the bad stock which so disastrously fails to adapt itself to its new environment, and in the sterility which so frequently ensues from the uterine lesions to which, as we have seen, the tuberculo-neurotic diathesis predisposes, there is placed another check upon the reproduction of these undesirable types; but it is time that artificial restrictions upon the procreation of the degenerate should be regarded as the imperative sequel to those improved conditions of health which conduce to their survival. In the crusade against tuberculosis which is now being waged, I perceive no reference to this important means of checking the ravages of this disease. Surely, if it be the duty of 
the State to give effect to measures which medical science shows to be requisite to lessen the incidence of disease, the obligation does not cease with the enforcement of merely sanitary measures. We do not improve the stud by stabling our horses under the most favourable of sanitary conditions, and our race should be perpetuated, as Dr. Harry Campbell showed the other day, by breeding from those only who would alone survive under more natural conditions.*

The legal sanction to procreate should be given only to couples whose physical fitness to bear healthy children is previously attested by a properly-constituted medical authority. This is a measure of preventive medicine the importance of which must increase in an inverse ratio to the decline in mortality from those diseases which are especially destructive of the degenerate, so far, at least, as that decline is dependent upon environmental changes, such as improved sanitation.

The law that degenerate types die out in a few generations is doubtless true under natural conditions; but where all effort is directed to preserving these types without at the same time insisting on their abstaining from bequeathing their infirmities to succeeding generations, a deterioration of the physique of the race is inevitable. The regulation of marriage is as much within the province of hygiene as is that of the other conditions upon which disease is dependent.

I should be misunderstood if for a moment it were believed that I am in any sense opposed to making the external conditions of health as perfect as may be ; but, to my mind, hygiene comprehends in its scope all that makes for the health of a community, and wholesome food, fresh air, and pure water are only next in importance to the breeding of virile men and healthy women.

Phthisis is a frightful scourge, and he would be brutal indeed who did not welcome the prospect of its disappearance. But the measures to be taken to accomplish that desirable end should not be such as to leave evils which, though immediately less obvious, are none the less real. And, if those measures are merely sanitary, if they do not comprehend all which the science of hygiene in its broader aspect demonstrates to be essential for the preservation of our racial physique and the maintenance of national health, the only effect will be the disappearance of diseases which, though rank and gross, are not more harmful than the subtle decay which will be the legacy of their effacement.

* The Lancet, September 10, 1898. 